\title{
ENUMERATION OF INDICES OF GIVEN ALTITUDE AND DEGREE
}

\author{
by I. M. H. ETHERINGTON \\ (Received 27th May 1959)
}

1. THIs note is a sequel to the article by Minc (2) on the same problem.

I described in (1) a notation for indices of powers in non-associative algebra, defined the degree $\dagger$ and altitude of a power or index, and observed that powers can be represented by bifurcating root-trees. For example, the power $x x . x$ is denoted $x^{2+1}$, with index $2+1$, and is represented by the tree $\downarrow$; the degree (the number of factors, or free knots in the tree) is 3 , and the altitude (the height of the tree) is 2. Multiplication being non-commutative or commutative, one maintains or ignores the distinction between left and right in the tree.

Let $a_{\delta}, p_{\alpha}, p(\alpha, \delta)$ denote the numbers of distinct indices of degree $\delta$, of altitude $\alpha$, of altitude $\alpha$ and degree $\delta$, in the non-commutative case; and let $b_{\delta}, q_{\alpha}, q(\alpha, \delta)$ be the corresponding numbers in the commutative case. I discussed the enumerations $a_{\delta}, b_{\delta}, p_{\alpha}, q_{\alpha}$ in (1), quoting some of the numerous writers who have considered $a_{\delta}, b_{\delta}$. In particular it is known that

$$
a_{\delta}=(2 \delta-2) ! /(\delta-1) ! \delta !,
$$

and that for $\delta=1,2,3, \ldots$

$$
\begin{aligned}
& a_{\delta}=1,1,2, \quad 5,14,42,132,429,1430,4862, \ldots \text {, } \\
& b_{\delta}=1,1,1,2, \quad 3, \quad 6, \quad 11, \quad 23, \quad 46, \quad 98, \ldots .
\end{aligned}
$$

Minc obtains two formulæ, giving $p(\alpha, \delta), q(\alpha, \delta)$ in terms of the same with smaller $\alpha$ and $\delta$, and calculates these numbers as far as $\alpha=4$. I shall show that explicit formulæ can be found for

$$
p(\alpha, \alpha+k), \quad p\left(\alpha, 2^{\alpha}-k\right), \quad q(\alpha, \alpha+k), \quad q\left(\alpha, 2^{\alpha}-k\right)
$$

for $k=1,2,3, \ldots$ in succession. These formulæ for small values of $k$ are given in $\S 6$. The initial formulæ in each group (and some others) are obvious by consideration of trees; the rest are derived via first order difference equations in $\alpha$.

The following results will be used. For any index of altitude $\alpha$ and degree $\delta$, $\alpha+1 \leqq \delta \leqq 2^{\alpha}$, so that

$$
p(\alpha, \delta)=q(\alpha, \delta)=0 \text { if } \delta \leqq \alpha \text { or }>2^{\alpha} ;
$$

$a_{\delta}=\sum_{\alpha=\left[\log _{2} \delta\right]}^{\delta-1} p(\alpha, \delta)=\sum_{\alpha=0}^{\kappa} p(\alpha, \delta), \quad b_{\delta}=\sum_{\alpha=0}^{\kappa} q(\alpha, \delta), \quad$ where $\kappa \geqq \delta-1$;

together with Minc's two formulæ for $p(\alpha, \delta), q(\alpha, \delta)$, some of his numerical results, and some of the values of $a_{\delta}, b_{\delta}$ quoted above.

$\uparrow$ Called potency by Minc.

E.M.S.-A 
2. We seek first a connexion between $p(\alpha, \alpha+k)$ and $p(\alpha+1, \alpha+1+k)$. Minc's first formula gives

$p(\alpha+1, \alpha+1+k)$

$$
=\sum_{d=\alpha+1}^{\alpha+k}\left[p(\alpha, d)\left\{2 \sum_{r=0}^{\alpha-1} p(r, \alpha+1+k-d)+p(\alpha, \alpha+1+k-d)\right\}\right] .
$$

Here, by (2), $\sum_{r=0}^{\alpha-1} p(r, \alpha+1+k-d)=a_{\alpha+1+k-d}$ provided that

$$
\alpha-1 \geqq \alpha+k-d, \text { i.e. } d-1 \geqq k, \quad \text { i.e. } \alpha \geqq k \text {. }
$$

Under the same condition $\alpha \geqq k$, we have $\alpha+1+k-d \leqq \alpha$ and therefore, by (1), $p(\alpha, \alpha+1+k-d)=0$. Thus if $\alpha \geqq k$

$$
p(\alpha+1, \alpha+1+k)=2 \sum_{d=\alpha+1}^{\alpha+k} a_{\alpha+1+k-d} p(\alpha, d) .
$$

Since $a_{1}=1$, the last term in this sum is $2 p(\alpha, \alpha+k)$. Hence $p(\alpha+1, \alpha+1+k)-2 p(\alpha, \alpha+k)$

$$
=2\left\{a_{k} p(\alpha, \alpha+1)+a_{k-1} p(\alpha, \alpha+2)+\ldots+a_{2} p(\alpha, \alpha+k-1)\right\} \quad(\alpha \geqq k) .
$$

If we assume that explicit formulæ are already known for $p(\alpha, \alpha+1)$, $p(\alpha, \alpha+2), \ldots, p(\alpha, \alpha+k-1)$, then we have a linear difference equation with constant coefficients from which to determine $p(\alpha, \alpha+k)$. The arbitrary constant in the solution is to be adjusted to give the right result when $\alpha=k$.

It is easily seen by consideration of trees that

$$
p(\alpha, \alpha+1)=2^{\alpha-1} \quad(\alpha \geqq 1) .
$$

Hence formulæ for $p(\alpha, \alpha+2), p(\alpha, \alpha+3), p(\alpha, \alpha+4), \ldots$ can be found in succession by solving difference equations, provided that the values of $p(2,4), p(3,6)$, $p(4,8), \ldots$ are known. (These values are $1,6,68, \ldots)$.

Calculation is facilitated by putting

$$
p(\alpha, \alpha+k)=f_{k}(\alpha) 2^{\alpha}
$$

The difference equation becomes

$$
\Delta f_{k}(\alpha) \equiv f_{k}(\alpha+1)-f_{k}(\alpha)=a_{k} f_{1}(\alpha)+a_{k-1} f_{2}(\alpha)+\ldots+a_{2} f_{k-1}(\alpha)
$$

so that

$$
f_{k}(\alpha)=\Delta^{-1}\left\{a_{k} f_{1}(\alpha)+a_{k-1} f_{2}(\alpha)+\ldots+a_{2} f_{k-1}(\alpha)\right\} \quad(\alpha \geqq k) .
$$

For illustration, let us suppose that the formulæ for $p(\alpha, \alpha+2)$ and $p(\alpha, \alpha+3)$ have been obtained (see $\S 6$ ), so that we have

$$
f_{1}(\alpha)=\frac{1}{2}, \quad f_{2}(\alpha)=\frac{1}{2} \alpha-\frac{3}{4}, \quad f_{3}(\alpha)=\frac{1}{4} \alpha^{2}-\frac{3}{2} .
$$

Then

$$
\begin{aligned}
f_{4}(\alpha) & =\Delta^{-1}\left\{5 \cdot \frac{1}{2}+2\left(\frac{1}{2} \alpha-\frac{3}{4}\right)+1\left(\frac{1}{4} \alpha^{2}-\frac{3}{2}\right)\right\} \\
& =\Delta^{-1}\left\{\frac{1}{4} \alpha(\alpha-1)+\frac{5}{4} \alpha-\frac{1}{2}\right\} \\
& =\frac{1}{12} \alpha(\alpha-1)(\alpha-2)+\frac{5}{8} \alpha(\alpha-1)-\frac{1}{2} \alpha+C,
\end{aligned}
$$


where $C$ is such that $f_{4}(4) 2^{4}=p(4,8)=68$. This gives $C=-\frac{13}{4}$, and finally

$$
\begin{gathered}
f_{4}(\alpha)=\frac{1}{24}\left(2 \alpha^{3}+9 \alpha^{2}-23 \alpha-78\right), \\
p(\alpha, \alpha+4)=\frac{1}{3}\left(2 \alpha^{3}+9 \alpha^{2}-23 \alpha-78\right) 2^{\alpha-3} \quad(\alpha \geqq 4) .
\end{gathered}
$$

Since $\Delta^{-1}$ raises the degree of a polynomial by 1 , the general result is of the form (3) ( $\alpha \geqq k)$, in which $f_{k}(\alpha)$ is a polynomial in $\alpha$ of degree $k-1$.

3. We seek next a difference equation for $p\left(\alpha, 2^{\alpha}-k\right)$. Minc's first formula gives

$$
\begin{aligned}
& p\left(\alpha+1,2^{\alpha+1}-k\right) \\
& \quad=\sum_{d=\alpha+1}^{2 \alpha+1-k-1}\left[p(\alpha, d)\left\{2 \sum_{r=0}^{\alpha-1} p\left(r, 2^{\alpha+1}-k-d\right)+p\left(\alpha, 2^{\alpha+1}-k-d\right)\right\}\right] .
\end{aligned}
$$

Now, by (1), $p(\alpha, d)=0$ if $d>2^{\alpha}$. Hence in the inner summation we may assume $d \leqq 2^{\alpha}$, so that

$$
2^{\alpha+1}-k-d \geqq 2^{\alpha+1}-k-2^{\alpha}=2^{\alpha}-k .
$$

Using this, and again using (1), we see that every term in the inner summation vanishes if

$$
2^{\alpha}-k>2^{\alpha-1} \text {, i.e. } 2^{\alpha-1}>k \text {, i.e. } \alpha \geqq 2+\left[\log _{2} k\right] \text {. }
$$

We assume this condition satisfied, and note that it implies that $\alpha \geqq 2$, hence $2^{\alpha-1} \geqq \alpha$; and since by (5) $2^{\alpha}-k \geqq 2^{\alpha-1}+1$, this implies that

$$
2^{\alpha}-k \geqq \alpha+1 \text {; }
$$

it can also be deduced that

$$
2^{\alpha}<2^{\alpha+1}-k-1
$$

(4) now reduces to

$$
p\left(\alpha+1,2^{\alpha+1}-k\right)=\sum_{d=\alpha+1}^{2^{\alpha+1}-k-1} p(\alpha, d) p\left(\alpha, 2^{\alpha+1}-k-d\right) .
$$

As already observed, the first factor in the summation is zero if $d>2^{\alpha}$; the second factor is zero if $2^{\alpha+1}-k-d>2^{\alpha}$, i.e. $d<2^{\alpha}-k$. Hence, in view of (6) and (7),

$$
p\left(\alpha+1,2^{\alpha+1}-k\right)=\sum_{d=2^{\alpha}-k}^{2^{\alpha}} p(\alpha, d) p\left(\alpha, 2^{\alpha+1}-k-d\right) .
$$

Now $p\left(\alpha, 2^{\alpha}\right)=1$, the only index of altitude $\alpha$ and degree $2^{\alpha}$ being $2^{\alpha}$. So the first and last terms in the summation (8) are both $p\left(\alpha, 2^{\alpha}-k\right)$. In the case $k=1$, this exhausts the summation, and we have the difference equation

$$
p\left(\alpha+1,2^{\alpha+1}-1\right)=2 p\left(\alpha, 2^{\alpha}-1\right) \quad(\alpha \geqq 2),
$$

whose solution $p\left(\alpha, 2^{\alpha}-1\right)=C .2^{\alpha}$ with initial condition $p(2,3)=2$ yields the formula

$$
p\left(\alpha, 2^{\alpha}-1\right)=2^{\alpha-1} \quad(\alpha \geqq 2) .
$$


If $k>1,(8)$ can be written

$$
p\left(\alpha+1,2^{\alpha+1}-k\right)-2 p\left(\alpha, 2^{\alpha}-k\right)=\sum_{d=2^{\alpha-k+1}}^{2^{\alpha}-1} p(\alpha, d) p\left(\alpha, 2^{\alpha+1}-k-d\right)
$$

or finally, putting $d=2^{\alpha}-k+r$,

$$
\begin{gathered}
p\left(\alpha+1,2^{\alpha+1}-k\right)-2 p\left(\alpha, 2^{\alpha}-k\right)=\sum_{r=1}^{k-1} p\left(\alpha, 2^{\alpha}-k+r\right) p\left(\alpha, 2^{\alpha}-r\right) \\
\left(k>1, \alpha \geqq 2+\left[\log _{2} k\right]\right) .
\end{gathered}
$$

If we write $p\left(\alpha, 2^{\alpha}-k\right)=u_{k}(\alpha)$, the equation is

$$
u_{k}(\alpha+1)-2 u_{k}(\alpha)=u_{k-1}(\alpha) u_{1}(\alpha)+u_{k-2}(\alpha) u_{2}(\alpha)+\ldots+u_{1}(\alpha) u_{k-1}(\alpha) .
$$

We know $u_{0}=1, u_{1}=2^{\alpha-1}$, and so by solving difference equations we can calculate $u_{2}, u_{3}, \ldots$ in succession. At each stage the arbitrary constant is to be adjusted to give the right result when $\alpha=2+\left[\log _{2} k\right]$. It can be shown inductively that the general form of the result is

$$
p\left(\alpha, 2^{\alpha}-k\right)=\text { a polynomial of degree } k \text { in } 2^{\alpha} \quad\left(\alpha \geqq 2+\left[\log _{2} k\right]\right) .
$$

4. By similar methods difference equations can be found for $q(\alpha, \alpha+k)$ and $q\left(\alpha, 2^{\alpha}-k\right)$. The details are rather tedious and $\mathbf{I}$ will merely quote the results which $I$ have obtained.

$$
\text { If } \begin{aligned}
\alpha \geqq k, \Delta q(\alpha, \alpha+k) & \equiv q(\alpha+1, \alpha+1+k)-q(\alpha, \alpha+k) \\
& =b_{k} q(\alpha, \alpha+1)+b_{k-1} q(\alpha, \alpha+2)+\ldots+b_{2} q(\alpha, \alpha+k-1) .
\end{aligned}
$$

Hence, for $\alpha \geqq k$,

$$
q(\alpha, \alpha+k)=\Delta^{-1}\left\{b_{k} q(\alpha, \alpha+1)+b_{k-1} q(\alpha, \alpha+2)+\ldots+b_{2} q(\alpha, \alpha+k-1)\right\}
$$

where the arbitrary constant is to be chosen to fit $q(k, 2 k)$; and $q(\alpha, \alpha+k)$ is a polynomial in $\alpha$ of degree $k-1$. The formulæ given in $\S 6$ (iii), going as far as $k=4$, proved thus for $\alpha \geqq k$, are found to be in fact true for $\alpha \geqq k-1$.

5. If $\alpha \geqq 2+\left[\log _{2} k\right]$, and if $q\left(\alpha, 2^{\alpha}-k\right)$ is denoted $v_{k}$, then

(i) if $k$ is odd,

$$
v_{k}=\Delta^{-1}\left\{v_{k-1} v_{1}+v_{k-2} v_{2}+\ldots+v_{(k+1) / 2} v_{(k-1) / 2}\right\} ;
$$

(ii) if $k$ is even,

$$
v_{k}=\Delta^{-1}\left\{v_{k-1} v_{1}+v_{k-2} v_{2}+\ldots+v_{k / 2+1} v_{k / 2-1}+\frac{1}{2} v_{k / 2}\left(v_{k / 2}+1\right)\right\} \text {. }
$$

The arbitrary constant is to be chosen to give the right result when $\alpha=2+\left[\log _{2} k\right]$. For $\alpha \geqq 2+\left[\log _{2} k\right], k \geqq 1, q\left(\alpha, 2^{\alpha}-k\right)$ is a polynomial in $\alpha$ of degree $k-1$.

\section{Conclusions}

$$
\text { (i) } \begin{aligned}
p(\alpha, \alpha+1) & =2^{\alpha-1} \\
p(\alpha, \alpha+2) & =(2 \alpha-3) 2^{\alpha-2} \\
p(\alpha, \alpha+3) & =\left(\alpha^{2}-6\right) 2^{\alpha-2} \\
p(\alpha, \alpha+4) & =\frac{1}{3}\left(2 \alpha^{3}+9 \alpha^{2}-23 \alpha-78\right) 2^{\alpha-3}
\end{aligned}
$$


(ii) $p\left(\alpha, 2^{x}\right)=1$

$$
\begin{aligned}
& p\left(\alpha, 2^{\alpha}-1\right)=2^{\alpha-1} \\
& p\left(\alpha, 2^{\alpha}-2\right)=2^{\alpha-2}\left(2^{\alpha-1}-1\right) \\
& p\left(\alpha, 2^{\alpha}-3\right)=\frac{1}{3} 2^{\alpha-2}\left(2^{2 \alpha-2}-3.2^{\alpha-1}+5\right)
\end{aligned}
$$

(iii) $q(\alpha, \alpha+1)=1$

$$
\begin{aligned}
& q(\alpha, \alpha+2)=\alpha-1 \\
& q(\alpha, \alpha+3)=\frac{1}{2}\left(\alpha^{2}-\alpha-2\right) \\
& q(\alpha, \alpha+4)=\frac{1}{6}\left(\alpha^{3}-\alpha-18\right)
\end{aligned}
$$

(iv) $q\left(\alpha, 2^{\alpha}\right)=1$

$$
\begin{aligned}
& q\left(\alpha, 2^{\alpha}-1\right)=1 \\
& q\left(\alpha, 2^{\alpha}-2\right)=\alpha-1 \\
& q\left(\alpha, 2^{\alpha}-3\right)=\frac{1}{2}\left(\alpha^{2}-3 \alpha+4\right) \\
& (\alpha \geqq 3) \text {. }
\end{aligned}
$$

\section{REFERENCES}

(1) I. M. H. Etherington, On non-associative combinations, Proc. Roy. Soc. Edin., 59 (1939), 153-162.

(2) H. Minc, Enumeration of indices of given altitude and potency, Proc. Edin. Math. Soc., 11 (1959), 207-209.

\section{Mathematical Institute}

THE UNIVERSITY

EDINBURGH 